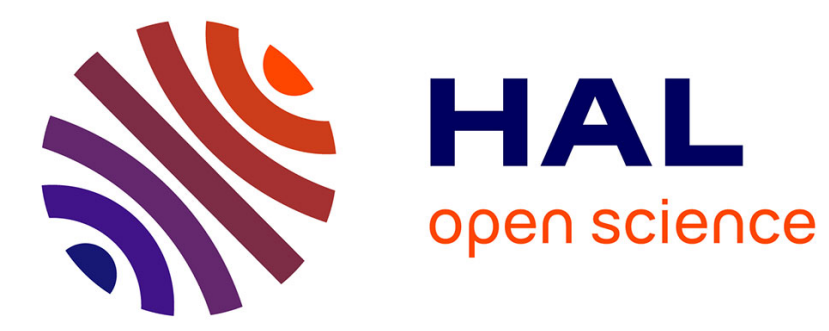

\title{
Quantum Hall states in inverted HgTe quantum wells probed by transconductance fluctuations
}

\author{
S. Mantion, Colin Avogadri, Sergey S. Krishtopenko, S. Gebert, Sandra \\ Ruffenach, Christophe Consejo, S. Morozov, N. N. Mikhailov, S. A. \\ Dvoretskii, Wojciech Knap, et al.
}

\section{To cite this version:}

S. Mantion, Colin Avogadri, Sergey S. Krishtopenko, S. Gebert, Sandra Ruffenach, et al.. Quantum Hall states in inverted $\mathrm{HgTe}$ quantum wells probed by transconductance fluctuations. Physical Review B, 2020, 102 (7), pp.075302. 10.1103/PhysRevB.102.075302 . hal-02919868

\section{HAL Id: hal-02919868 https://hal.science/hal-02919868}

Submitted on 1 Dec 2020

HAL is a multi-disciplinary open access archive for the deposit and dissemination of scientific research documents, whether they are published or not. The documents may come from teaching and research institutions in France or abroad, or from public or private research centers.
L'archive ouverte pluridisciplinaire HAL, est destinée au dépôt et à la diffusion de documents scientifiques de niveau recherche, publiés ou non, émanant des établissements d'enseignement et de recherche français ou étrangers, des laboratoires publics ou privés. 


\title{
Quantum Hall states in inverted HgTe quantum wells probed by transconductance fluctuations
}

\author{
S. Mantion, ${ }^{1,2}$ C. Avogadri, ${ }^{1}$ S. S. Krishtopenko, ${ }^{1}$ S. Gebert,${ }^{3}$ S. Ruffenach, ${ }^{1}$ C. Consejo, ${ }^{1}$ S. V. Morozov,${ }^{4,5}$ \\ N. N. Mikhailov, ${ }^{6,7}$ S. A. Dvoretskii,,${ }^{6,7,8}$ W. Knap,${ }^{1,9}$ S. Nanot,${ }^{1}$ F. Teppe, ${ }^{1}$ and B. Jouault ${ }^{1}$ \\ ${ }^{1}$ Laboratoire Charles Coulomb (L2C), UMR 5221, \\ CNRS-University of Montpellier, 34095 Montpellier, France. \\ ${ }^{2}$ CEMES-CNRS, UPR 8011, Universitd́e Toulouse, \\ 29 rue Jeanne Marvig, BP 94347, 31055 Toulouse. \\ ${ }^{3}$ Institut d'Electronique et des Systèmes, CNRS-University of Montpellier, 34000 Montpellier, France \\ ${ }^{4}$ Institute for Physics of Microstructures RAS, GSP-105, Nizhni Novgorod 603950, Russia. \\ ${ }^{5}$ Lobachevsky State University of Nizhni Novgorod, \\ prospekt Gagarina 23, Nizhni Novgorod 603950, Russia. \\ ${ }^{6}$ Institute of Semiconductor Physics, Siberian Branch, Russian Academy of Sciences, \\ pr. Akademika Lavrent'eva 13, Novosibirsk, 630090 Russia. \\ ${ }^{7}$ Novosibirsk State University, Pirogova st. 2, 630090 Novosibirsk, Russia. \\ ${ }^{8}$ Tomsk State University, Tomsk, 634050, Russia. \\ ${ }^{9}$ CENTERA Laboratories, Institute of High Pressure Physics PAS, Warsaw 01-142, Poland.
}

(Dated: July 2, 2020)

\begin{abstract}
We investigated quantum Hall states in an inverted HgTe quantum well (QW) close to the critical thickness using transconductance fluctuation (TF) measurements. In the conduction band, several integer quantum Hall states were observed, corresponding to filling factor $\nu=1,2,3,4$. For magnetic fields above $2 \mathrm{~T}$, quantum Hall states $\nu=0$ were observed in the normal gap. These observations agreed well with the previous studies of quantum Hall states on GaAs QWs and graphene. Interestingly, TFs corresponding to anomalous positive filling factor $\nu$ were clearly observed in the valence band. We attribute the emergence of those TFs to the localization and charging of the heavy holes located in the side maxima of the valence band.
\end{abstract}

\section{INTRODUCTION}

It is well known that HgTe quantum wells (QW) can act as either a band insulator (BI) or a non-trivial topological insulator (TI) [1], depending on quantum well thickness [2], temperature [3], and hydrostatic pressure [4]. For gapped HgTe QWs, the spectrum of the conduction band $(\mathrm{CB})$ is parabolic at small momentum and becomes linear at large $k$. On the contrary, the spectrum of the valence band (VB) is non-monotonic with side maxima arising at $k>0$. So far, only a few publications have investigated the VB of these QWs [5-10]. Although they provide a good qualitative description of the $\mathrm{VB}$, they fail to provide a quantitative examination with good agreement to existing theories. For instance, the experimentally determined hole effective masses and the position of the side maxima in the $\mathrm{VB}$ do not correspond to the theoretical prediction [8], and the importance of spin-orbit coupling in the VB was only recently detected [10].

A crucial way of analyzing the band structure is to probe the dispersion of the Landau levels (LLs) under a magnetic field. By doing so, a remarkable quantum Hall (QH) plateau has been observed when the Fermi energy is in the VB [11]. It is not clearly demonstrated, however, that the quantum plateau is stabilized by some parts of the VB acting as a charge reservoir. In fact, the charge transfer between interface states and a twodimensional electron gas (2DEG) is often considered as a complementary process to the well-known localization mechanism accounting for the finite width of the quantum Hall plateaus [12]. Such reservoir-related effects have also been observed in graphene on $\mathrm{SiC}$ [13-15].

In this work, we exhibit the interplay between the charge transfer and localization in the quantum Hall regime for inverted HgTe QWs. Recent studies have shown that transconductance measurements may reveal microscopic information on 2DEGs, even when macroscopic devices are measured $[16,17]$. Moreover, the transconductance, i.e. the ratio of the change in drain current to the change in gate voltage, can capture the phenomena of charge localization in the QH regime. Such charge localization was previously observed in various 2DEGs, e.g. GaAs QWs, and monolayer and bilayer graphene. Transconductance fluctuations appearing in the (carrier density-magnetic field) plane can even reveal very fragile fractional quantum Hall states that only locally form although these states do not appear in conventional magnetotransport measurements. Therefore, transconductance measurements in the $\mathrm{QH}$ regime on HgTe QWs can help to understand the nature of localization when the charge is partitioned between different quasiparticles.

Interestingly, a single-electron picture is expected in $\mathrm{HgTe}$ QWs because of the very high permittivity $\epsilon$ [18]. Moreover, an external potential is almost perfectly screened due to this high $\epsilon$ value. Therefore, HgTe QWs are excellent candidates to explore the universal theories of the QHE, e.g. the semicircle model [19], or the theory of the critical exponent [20] as these theories are based on 
noninteracting electrons moving into a weak long-range potential. On the other hand, experimental studies have shown poor agreement with these theories [21]. Hence, the transconductance in these systems can reveal if the single-particle picture is relevant or if charging effects prevail.

\section{MODEL OF DISORDER AND LOCALIZATION}

Fluctuations in the QH regime were first observed in the magnetoresistance of microscopic Si transistors [22] and graphene devices [23, 24]. Using single-electron transistors, similar fluctuations were observed in the local compressibility of 2DEGs, first in GaAs QWs [25] and later in graphene [26]. Recently, the same type of fluctuations was observed in the transconductance of graphene [16] and bilayer graphene [17], where fractional quantum states were identified. These experiments showed that the fluctuations moved parallel to lines corresponding to integer or fractional filling factor $\nu$ in the (density, magnetic field) plane and were attributed to charging effects and localization. The underlying model is detailed below.

Each QW structure has a specific disorder configuration which gives rise to a disorder potential $V_{\text {bare }}(\mathbf{r})$ in its plane. This potential forms hills and valleys and is independent of the magnetic field $B$ and charge density $n$. In a noninteracting picture, $V_{\text {bare }}(\mathbf{r})$ is the effective potential $V_{\text {eff }}(\mathbf{r})$ acting on the 2DEG. In the quantum Hall regime, the fluctuations of the conductance, transconductance, and the local compressibility are due to transmission through specific localized states, which follow equipotential lines of the disorder potential $V_{\text {bare }}(\mathbf{r})$. The energy of these localized states is usually nonmonotonic with $B$ and does not correspond to a straight line in the (density, $B$ ) plane, contradicting with the experimental observations [16, 17, 22-26].

Let us now take into account nonlinear screening and charge localization. We assume that the electron gas is infinitely compressible. The charge density reorganizes to screen the external potential. The relation between $V_{\text {bare }}$ and the charge density profile $n_{\text {scrn }}$ is given by the Poisson equation: $\nabla^{2} V_{\text {bare }}(\mathbf{r}, z)=e n_{\text {scrn }}(\mathbf{r}) \delta(z) / \epsilon$, where $-e$ is the electron charge. Now, in high magnetic fields, LLs appear and the local charge $n(\mathbf{r})$ is partitioned between them:

$$
n(\mathbf{r})=\delta n(\mathbf{r})+N n_{\max }
$$

where $n_{\max }=e B / h$ is the LL degeneracy, $\delta n(\mathbf{r})$ the extra charge appearing on the upper LL close to $E_{F}$, which is either partially populated $\left(n_{\max }>\delta n>0\right)$ or depopulated $\left(-n_{\max }<\delta n<0\right) . N$ is the Chern number of the 2DEG, a positive (negative) integer, which corresponds to the number of LLs of the CB (of the VB) below (above) the Fermi energy $E_{F}$. As the last term of Eq. 1 does not depend on $\mathbf{r}$ and hence cannot screen the disorder, the density profile $\delta n(\mathbf{r})$ should equal $n_{\text {scrn }}(\mathbf{r})$ to minimize the electrostatic energy and screen $V_{\text {bare }}(\mathbf{r})$. However, as $\delta n(\mathbf{r})$ has lower and upper bounds due to the LL degeneracy, $\delta n(\mathbf{r})$ may depart significantly from $n_{\text {scrn }}(\mathbf{r})$. The screening is then incomplete, and the potential tears the 2DEG into pieces. Some regions become metallic with a flat screened potential. Others become insulating where the LL is either empty or full. In the metallic regions, Coulomb blockade comes into play and gives rise to fluctuations in the conductance, transconductance, and local compressibility.

To illustrate this situation, let us start with $N$ filled LLs while the $(N+1)$-th LL is completely empty. The average carrier density $n$ is then $n=N n_{\max }$, and there is no mobile charge or screening. Increasing $n$, additional electrons appear in the $(N+1)$-th LL and accumulate in the low-energy regions of the potential, separated by an incompressible insulating region. As screening is possible only in the populated low-energy regions, $V_{\text {bare }}(\mathbf{r})$ is imperfectly screened, and the effective potential $V_{\text {eff }}(\mathbf{r})$ is composed of insulating hills $(\delta n(\mathbf{r})=0)$ and metallic flat lakes $(\delta n(\mathbf{r})>0)$. The shape of $V_{\text {eff }}(\mathbf{r})$ governs the conductance and depends only on the average carrier density $\delta n$. Thus, we can assume that the observed fluctuations correspond to curves of constant $\delta n$ in the $(n, B)$ plane. In the presence of a metallic gate, we have $n=\left(C_{g} / e\right) V_{g}$, where $V_{g}$ is the gate voltage and $C_{g}$ is the geometric capacitance of the gate. From the relation: $\frac{C_{g}}{e} V_{g}=\delta n+N n_{\max }$, it follows that the curves of the fluctuations, i.e. the curves of constant $\delta n$, correspond in the $\left(V_{g}, B\right)$ plane to straight lines of slope

$$
\frac{d V_{g}}{d B}=N \times \frac{e^{2}}{h} \times \frac{1}{C_{g}} .
$$

We end up with the same result if we start with $N$ completely filled LLs and progressively depopulate the upper LL $(\delta n<0)$. Remarkably, the formation of the screened potential $V_{\text {eff }}(\mathbf{r})$ is determined solely by the density deviation from the completely full LLs, i.e. $\delta n$, and not by the total density $n$.

\section{SAMPLE PRESENTATION}

The heterostructure presented in this work has been used in our recent reports on the existence of a temperature-driven phase transition [3] and the observation of enhanced stability of quantum Hall plateaus [11]. The structure was grown by molecular beam epitaxy (MBE) on a [013]-oriented semi-insulating GaAs substrate with a relaxed CdTe buffer. The $\mathrm{HgTe}$ QW of $6.6 \mathrm{~nm}$ width was embedded in $40-\mathrm{nm} \mathrm{Cd}_{0.65} \mathrm{Hg}_{0.35} \mathrm{Te}$ barriers. A 40-nm CdTe cap layer was deposited on top of the structures. The barriers from both sides of the QW were selectively doped with indium. After MBE growth, 100-nm $\mathrm{SiO}_{2}$ and 200-nm $\mathrm{Si}_{3} \mathrm{~N}_{4}$ dielectric layers were deposited on top of the structure by a plasmochem- 


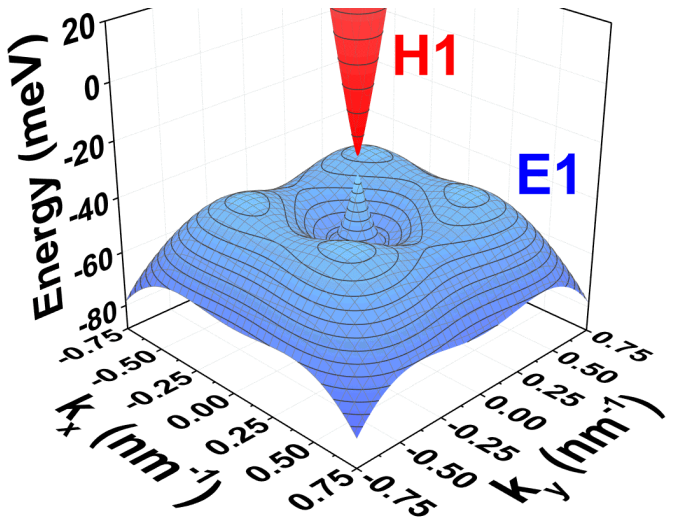

FIG. 1. Band structure of our sample at $T=260 \mathrm{mK}$ calculated on the basis of the 8 -band $\mathbf{k} \cdot \mathbf{p}$ Hamiltonian [4]. The $\mathrm{VB}$ and the $\mathrm{CB}$ are shown in blue and red, respectively.

ical method. The gated Hall bar has a total length of $650 \mu \mathrm{m}$ and a total width of $50 \mu \mathrm{m}$.

Fig. 1 shows the expected band structure for the studied QW, as given by an 8-band $\mathbf{k} \cdot \mathbf{p}$ model. The CB shows a quasi-linear dispersion. There is a small topological gap of $8 \mathrm{meV}$ between the $\mathrm{CB}$ and VB. The VB also shows a quasi-linear dispersion in the center of the Brillouin zone. At approximately $\left|k_{x}\right|=\left|k_{y}\right|=0.4 \mathrm{~nm}^{-1}$, 4 local side maxima can be distinguished in the $E_{1} \mathrm{VB}$. They appear because of the zincblende structure of the crystal. In the following, we name "light holes" the carriers located in the central part of the $E_{1} \mathrm{VB}$, and "heavy holes" the carriers located in the 4 local maxima.

\section{MEASUREMENTS}

\section{A. Magnetoresistances}

Fig. 2(a,b) show the transverse and the longitudinal magnetoresistances as a function of the magnetic field for a gate voltage $V_{g}=-3 \mathrm{~V}$ at different temperatures. The sign of the Hall resistance indicates that the Fermi energy is in the VB. Fig. 2(c) shows the experimental Hall carrier density as a function of the gate voltage. The Fermi energy is within the topological gap at around $V_{g} \simeq-1.6 \mathrm{~V}$. At $V_{g}>-1.6 \mathrm{~V}$, when the Fermi energy $E_{F}$ lies in the $\mathrm{CB}$, the gate voltage dependence of the Hall concentration $n_{H}$ follows a slope corresponding precisely to the expected geometric capacitance $C_{g}$ : $d n_{H} / d V_{g}=C_{g} / e \simeq 9.1 \times 10^{10} \mathrm{~cm}^{-2} / V$. The situation is completely different for $V_{g}<-1.6 \mathrm{~V}$, when the Fermi energy is located in the VB. The Hall concentration, then, follows a slope $d n_{H} / d V_{g} \simeq 1.0 \times 10^{10} \mathrm{~cm}^{2} / \mathrm{V}$, almost 10 times smaller than the slope expected from the geometric capacitance. We attribute this anomalous slope, already observed in Refs. 10 and 27, to the coexistence of light and heavy holes. The heavy holes have low mobility and do not contribute to the classical Hall effect in which only
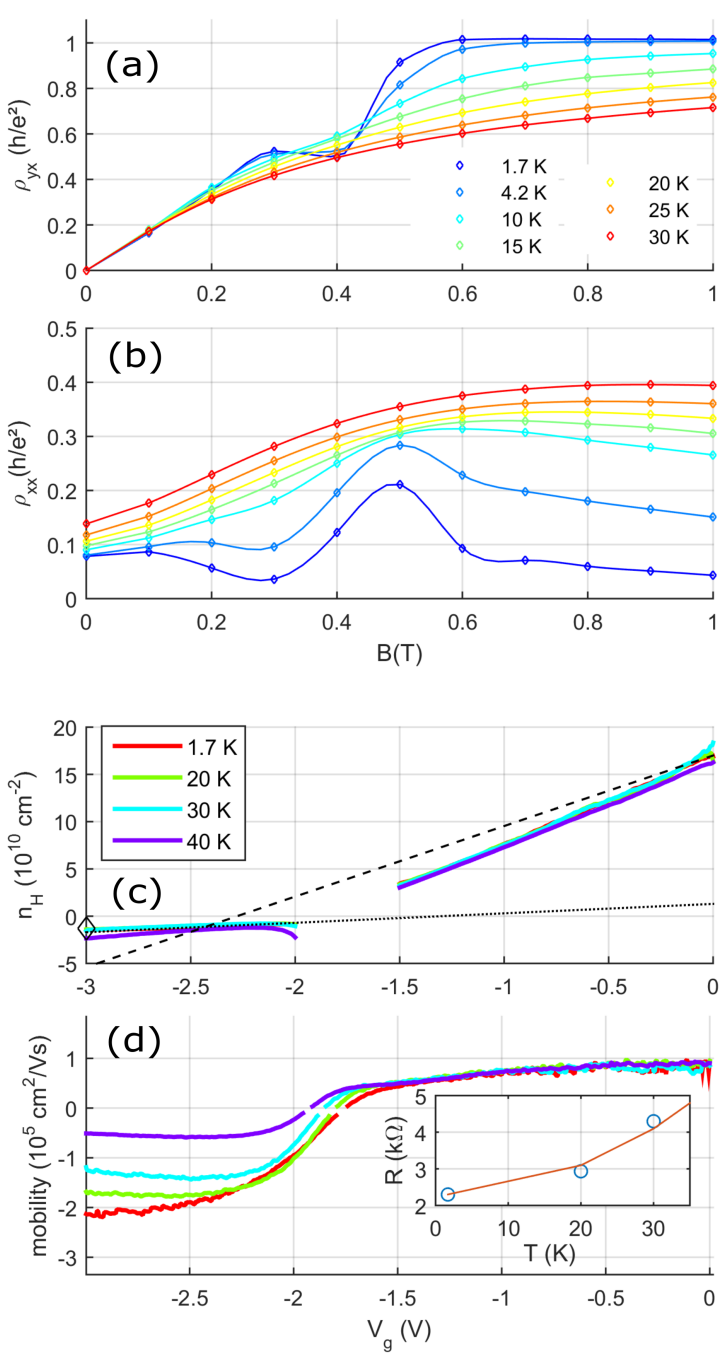

FIG. 2. (a) Transverse and (b) longitudinal magnetoresistances at several temperatures $T=1.7 \mathrm{~K}, 4.2 \mathrm{~K}, 10 \mathrm{~K}, 15 \mathrm{~K}$, $20 \mathrm{~K}, 25 \mathrm{~K}$ and $30 \mathrm{~K}$ for $V_{g}=-3 \mathrm{~V}$. (c) Hall concentration $n_{H}$ as a function of the gate voltage at $T=1.7 \mathrm{~K}, 20 \mathrm{~K}, 30 \mathrm{~K}$ and $40 \mathrm{~K}$. The diamond at $V_{g}=-3 \mathrm{~V}$ corresponds to the concentration deduced from the $\mathrm{SdH}$ period at $T=1.7 \mathrm{~K}$. (d) Mobility as a function of the gate voltage, at the same temperatures as in (c). The inset is a fit of the resistance $R=1 /\left(n_{H} e \mu\right)$ (open symbols) at $V_{g}=-3 \mathrm{~V}$ with a quadratic function, $R(B)-R(0) \propto T^{2}$ (solid line).

light holes participate. However, the heavy holes have a large density of states and pin the light hole density at an almost constant Fermi energy $E_{F}$, so the $n_{H}$ density becomes almost independent of $V_{g}$.

The coexistence of different kinds of holes is confirmed by the mobility analysis. As observed in Fig. 2(d), the Hall mobility in the VB is three times higher than the mobility of the electrons in the $\mathrm{CB}$ for the same carrier concentration at $T=1.7 \mathrm{~K}$. We attribute this effect to the presence of the heavy holes, which have a high density and efficiently screen the ionized impurities. The Hall 


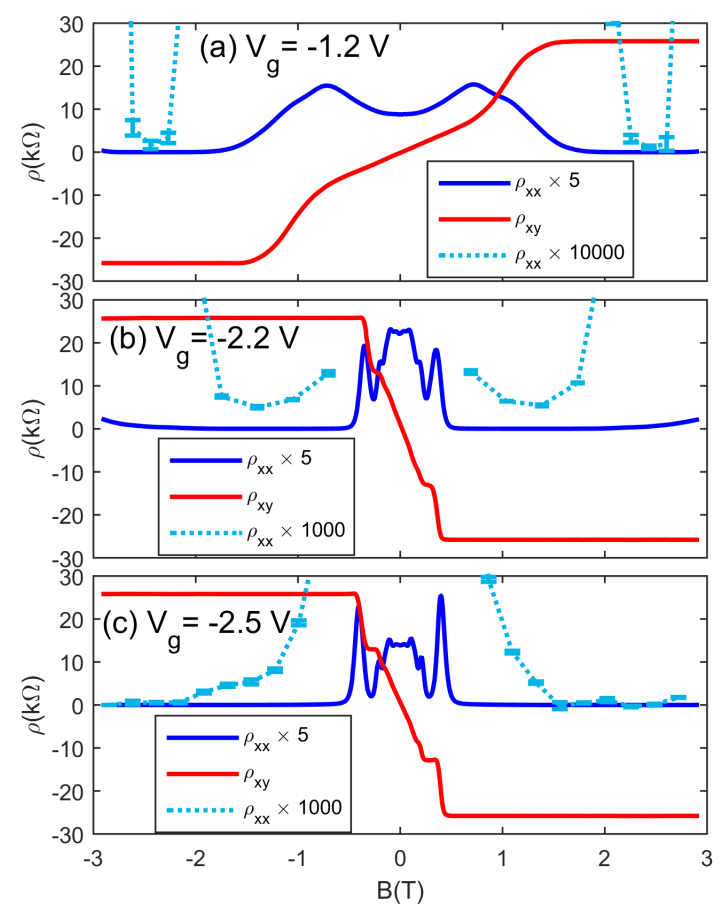

FIG. 3. Longitudinal (blue lines) and transverse (red lines) magnetoresistances measured at $1.7 \mathrm{~K}$ for 3 gate voltages: (a) $V_{g}=-1.2 \mathrm{~V}$, (b) $V_{g}=-2.2 \mathrm{~V}$ and $V_{g}=-2.5 \mathrm{~V}$. The Fermi level lies in the CB in (a) and in the VB in (b,c). The cyan lines correspond to a magnified view of the longitudinal resistances.

mobility then corresponds to the light hole mobility [27, $28]$. The presence of two types of carriers is sustained by the temperature dependence of the hole mobility, which decreases roughly as $1 / T^{2}$ below $T \leq 30 \mathrm{~K}$, as seen in the inset of Fig. 2(d). This suggests that scattering between light and heavy holes is the limiting factor for the light hole mobility. Indeed, the coexistence of light and heavy holes results in their mutual scattering via the Baber mechanism $[29,30]$, which is proportional to $T^{2}$. By contrast, the Hall mobility in the CB depends weakly on temperature below $40 \mathrm{~K}$, confirming the presence of only one type of carrier.

Let us now analyze the situation in a quantizing magnetic field. Fig. 3(a) shows the longitudinal and the transverse magnetoresistance of the Hall bar at $T=1.7 \mathrm{~K}$ and $V_{g}=-1.2 \mathrm{~V}$, when $E_{F}$ is in the CB. The plateau corresponding to Chern number $N=1$ is visible only after $B=1.5 \mathrm{~T}$. The minimum of the longitudinal resistance in the quantum regime appears around $B=2 \mathrm{~T}$, and the residual resistance is less than $0.1 \Omega$. Fig. 3(b) shows the magnetoresistances at $V_{g}=-2.2 \mathrm{~V}$ when $E_{F}$ is now in the VB. The Shubnikov-de Haas oscillations are well visible and give a concentration $n_{S d H} \simeq n_{H}$. At $B>0.5 \mathrm{~T}$, the $\mathrm{QH}$ effect emerges and a large plateau corresponding to Chern number $N=-1$ appears. Its very large width is reminiscent of the large plateaus $N=2$ appearing in graphene on $\mathrm{SiC}[15,31]$. This suggests

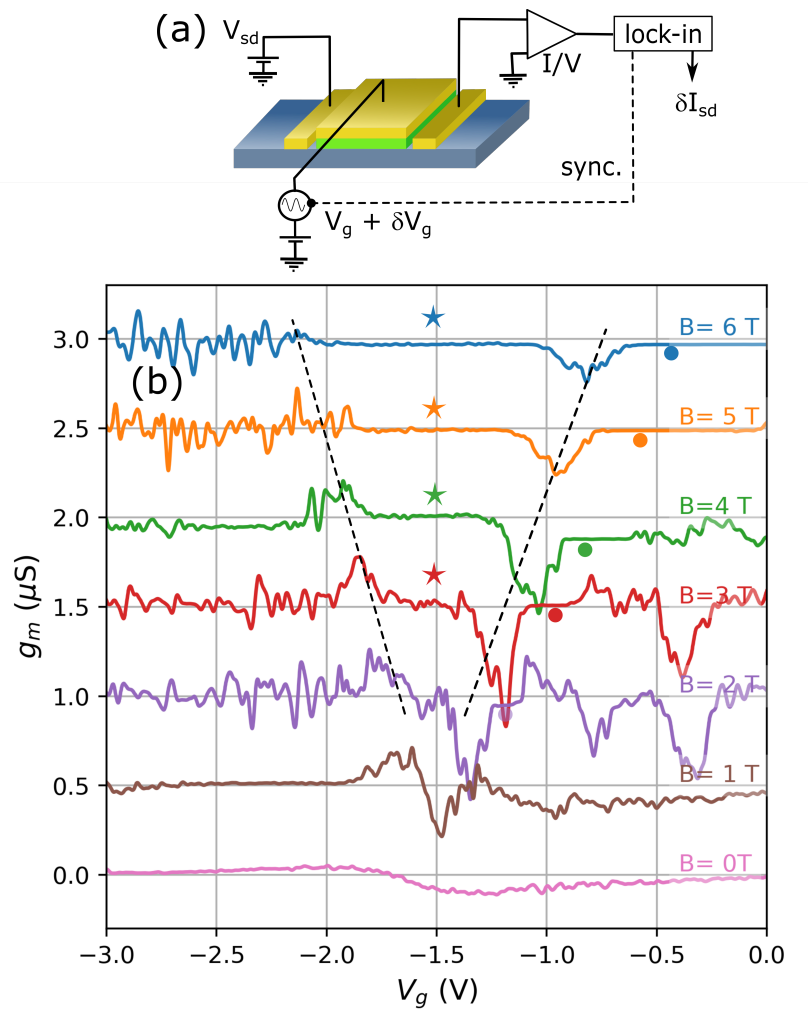

FIG. 4. (a) Scheme of the transconductance setup. (b) Transconductance $g_{m}$ as a function of gate voltage $V_{g}$, at $T=260 \mathrm{mK}$ and from $B=0 \mathrm{~T}$ up to $B=6 \mathrm{~T}$. The stars and plain circles indicate the positions of the $\nu=0$ and $\nu=1$ regions, respectively. The two black dashed lines indicate the position of the two zero-mode LLs.

that charge transfer takes place between the light holes and some reservoir, presumably the heavy holes located in the side maxima.

The residual conductivity of the heavy holes must be extremely small in the $\mathrm{QH}$ regime. In Fig. $3 \mathrm{~b}, \rho_{x x}$ has a minimum $\rho_{x x}^{\min } \simeq 5 \Omega$ at $B \simeq 1.5 \mathrm{~T}$ on the $N=-1$ plateau. Let us assume that all the residual resistivity $\rho_{x x}^{\min }$ is due to the presence of heavy holes. Then, the residual conductivity $\sigma_{h h}$ of heavy holes can be evaluated as $\sigma_{h h}=\rho_{x x}^{\min } / R_{K}^{2}$, where $R_{K}=h / e^{2}$.

The heavy hole concentration $n_{h h} \simeq 2 \times 10^{10} \mathrm{~cm}^{-2}$ can be deduced from the difference between the concentration from the estimated gate capacitance (the black dashed line in Fig. 2(c)) and the measured light hole concentration $n_{H}$. We obtain a mobility $\mu_{h h}=\sigma_{h h} / n_{h h} e \simeq$ $2 \mathrm{~cm}^{2} / \mathrm{Vs}$, much smaller than the mobility $\mu_{h h} \simeq(2-$ 3) $\times 10^{3} \mathrm{~cm}^{2} /$ Vs calculated in Ref. 10 for a similar structure. The same analysis can be done at $V_{g}=-2.5 \mathrm{~V}$. At this gate voltage, as shown in Fig. 3(c), the residual resistivity in the $\mathrm{QH}$ regime shifts to higher magnetic fields $B \geq 2.5 \mathrm{~T}$, as expected if the carrier concentration increases, but also drops by one order of magnitude, $\rho_{x x}^{\min } \leq 0.1 \Omega$. This gives extremely small mobility for the heavy holes, $\mu_{h h} \simeq 0.02 \mathrm{~cm}^{2} /$ Vs. These very small 
mobilities suggest that localization takes place for both light holes and heavy holes in the $\mathrm{QH}$ regime.

\section{B. Transconductance}

The transconductance measurements were performed in a two-probe configuration at a base temperature of $260 \mathrm{mK}$. A constant DC bias voltage $V_{s d}=2 \mathrm{mV}$ was applied between the source and drain. A small AC voltage $\delta V_{g}$ of $2 \mathrm{mV}$ (corresponding to a carrier density variation of 2 electrons per micrometer square) and frequency $71 \mathrm{~Hz}$ was superimposed to a chosen DC gate voltage $V_{g}$ and the corresponding source-drain current $\delta I_{s d}$ was detected at fixed $V_{g}$ with a lock-in amplifier. Then the transconductance $g_{m}=\delta I_{s d} / \delta V_{g}$ was measured while the gate voltage $V_{g}$ was swept between $-3 \mathrm{~V}$ and $0 \mathrm{~V}$ and the magnetic field $B$ between 0 and $6 \mathrm{~T}$. Three different pairs of probes were tested, and similar results were obtained for all pairs. In what follows we present the results obtained for one of these pairs.

Fig. 4 shows the transconductance as a function of the gate voltage $V_{g}$ at $T=260 \mathrm{mK}$ and for different magnetic fields from $B=0 \mathrm{~T}$ up to $B=6 \mathrm{~T}$. The curves are completely different from what is observed in the usual $\mathrm{QH}$ regime in a four-probe configuration, as in Fig. 3. The curves in Fig. 4 reveal a rich pattern of fluctuations, which are not noise and are fully reproducible. Some regions indicated by stars and circles appear flat and have been identified as the quantum plateaus $N=0$ and $N=$ 1. In other regions, large bumps and dips appear with a width of $200 \mathrm{mV}$. These features result from the large variation in conductance when the Fermi level crosses an LL. The two dashed black lines correspond to the two of these features, which are identified as the two zero-mode LLs. Finally, in other regions, rapid fluctuations appear with a smaller width $\delta V_{g} \simeq 50 \mathrm{mV}$.

Transconductance measurements such as the one shown in Fig. 4 have been repeated between $B=0 \mathrm{~T}$ and $B=6 \mathrm{~T}$ with a step size as small as $10 \mathrm{mT}$. Figure 5 displays a color map of the transconductance $g_{m}\left(V_{g}, B\right)$. The background of the transconductance has been subtracted to enhance the visibility of the rapid fluctuations, exposing a rich set of lines.

\section{Conduction band}

First, we analyze the map corresponding to the $\mathrm{CB}$ $H_{1}$, for $V_{g}>-1.6 \mathrm{~V}$. The TFs are clearly visible as lines in the $\left(V_{g}, B\right)$ plane, whose slopes correspond roughly to integer filling factors. The quantization of the TF slope is apparent along the line of integer filling factor $\nu=0$, $\nu=1$ and $\nu=2$, indicated with white solid lines in Fig. 5.

To confirm the quantization of the TF slopes, we used a more quantitative analysis. We calculated the correlation function $\mathcal{C}(\nu)=\sum D_{i j} D_{k l} \delta_{\nu}$, where $D_{i j}$ corresponds to a data point at gate voltage $V_{i}$ and magnetic field $B_{j}$ located within a chosen analysis window. A pair of data points $\left(D_{i j}, D_{k l}\right)$ contributes to the correlation $\mathcal{C}(\nu)$ only if the delta function $\delta_{\nu}=1, i$. e., if the line connecting the two points has a slope, which corresponds to $\nu$. A plot of $\mathcal{C}(\nu)$ then highlights those filling factors, which correspond to the preferential slopes of the TFs in the analysis window. A similar procedure has been previously applied for data recorded on monolayer and bilayer graphene [16, 17].

Figure $5(\mathrm{~b})$ plots the correlation functions $\mathcal{C}(\nu)$ for seven different analysis windows chosen exclusively in the region corresponding to the $\mathrm{CB}$. These analysis windows are indicated in Fig. 5(a) by solid open rectangles of the same color as their corresponding correlation function. The $\mathcal{C}(\nu)$ peaks in Fig. 5(b) reveal clearly the integer quantum states $\nu=0,1,2,3$, and 4 . There is a small deviation (10\%) of the correlation peaks with respect to their expected position because of the error when determining the geometric capacitance. Therefore, the analysis of the TFs observed in the CB validates the model presented above, based on localization, charging, and partial screening of the disorder.

\section{Valence band}

Let us now analyze the TFs visible in the VB. In Fig. 5(a) the thick black dotted polygon encloses the region corresponding to the $N=-1$ plateau, as determined by the experimental Hall conductivity. In this region, different regimes are clearly distinguishable. The analysis of the TFs reveals the presence of integer QH states $\nu=-1$ close to the edges of this region.

These states can be seen in the analysis windows indicated by the dashed black and blue rectangles. The corresponding correlation functions of these windows are reported in Fig. 5(c), where the peak of $\mathcal{C}(\nu)$ at $\nu=-1$ is easily identified.

Beyond these expected TFs, deep inside the $N=-1$ plateau, remarkable TFs can be determined. For $1.2 \mathrm{~T}$ $<B<3.0 \mathrm{~T}$, bunches of TFs of identical positive slopes are immediately noticed by eyes. A complete correlation analysis has been performed, and some representative correlation functions are reported in Fig. 5(c), for 3 analysis windows indicated by dashed red, magenta, and yellow rectangles in Fig. 5(a). Several correlation peaks are found at positive $\nu$ 's. Correlation peaks appear mainly around $\nu=0$ (all three dashed regions), $\nu=1$ (magenta and yellow dashed regions), $\nu=3-6$ (all three regions again). Even if correlation peaks and line bunches are easily identified, the superimposition of the correlation peaks in Fig. 5(c) suggest that the slopes of the TFs are not fully quantized, in sharp contrast to what is observed in Fig. 5(b). An intriguing trend is that the slope of the TFs visible in the range $B=1.2-3 \mathrm{~T}$ increases when $V_{g}$ increases. Indeed, $\mathcal{C}$ has its maximum at $\nu \simeq 3,4,5$ for the analysis regions corresponding to the yellow, ma- 

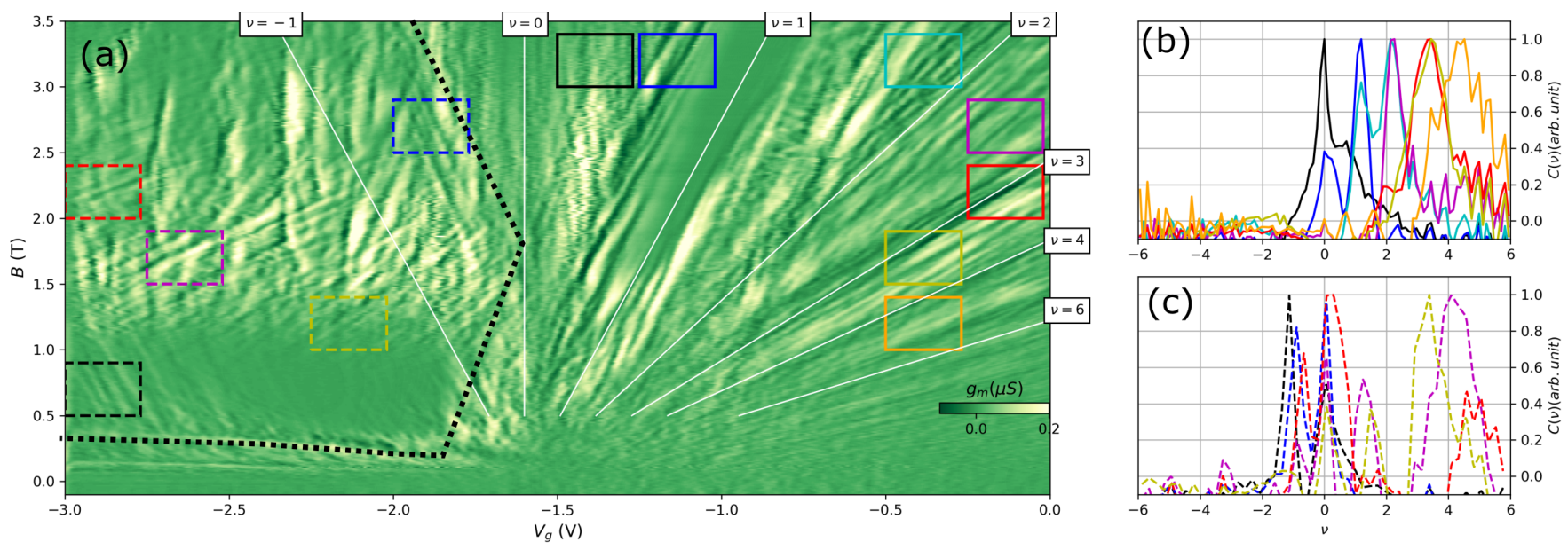

FIG. 5. Color map of the transconductance $g_{m}$ in the $\left(V_{g}, B\right)$ plane. The solid white lines correspond to integer filling factors. The dotted black line encloses the $N=-1$ plateau as determined from the Hall conductivity. (b) Correlation function $\mathcal{C}(\nu)$ for 7 analysis windows in the $\left(V_{g}, B\right)$ plane, located in the CB and indicated by solid colored rectangles in (a). (c) Correlation function $\mathcal{C}(\nu)$ for 5 analysis windows in the $\left(V_{g}, B\right)$ plane, located in the VB and shown as dashed colored rectangles in (a).

genta, and red dashed regions, respectively, which are centered around increasing $V_{g} \simeq-2.9,-2.6,-2.1 \mathrm{~V}$.

Finally, at $B>3.0 \mathrm{~T}$, the situation clarifies and all the TF slopes roughly align along $\nu=0$ (constant $V_{g}$ ). Similar results were reproduced for all three pairs of contacts analyzed on this device.

\section{INTERPRETATION}

\section{A. Estimation of the disorder potential}

The quantization of the TF slope in the CB allows us to use the model of screened disorder and localization as described above. The screening is, however, neither perfect nor linear. Even in the center of the LLs, TF lines of different slopes overlap, indicating that the network of percolating puddles is still present. This overlap is well visible in Fig. 5(a) in the blue (cyan) solid analysis window, where TFs of slopes $\nu=0,1(\nu=1,2)$ coexist. The correlation function in this window has also two pronounced peaks at $\nu=0$ and $\nu=1(\nu=1$ and $\nu=2)$. Additional measurements have shown that the TFs still overlap in the center of the two first LLs up to at least $B_{\max }=6 \mathrm{~T}$. Following Refs. 16 and 25 we get the lower bound of the carrier density $\Delta n_{d}$ needed to completely screen the bare disorder potential: $\Delta n_{d} \geq B_{\max } e / h \simeq 1.4 \times 10^{11} \mathrm{~cm}^{-2}$. This value is comparable to that observed for graphene on $\mathrm{SiO}_{2}[16]$ and 10 times larger than the value observed in GaAs QWs [25].

\section{B. Fermi level pinning in the valence band}

Before discussing the TF fluctuations, let us first focus on the remarkable width of the $N=-1$ plateau, as enclosed by the black dashed line in Fig. 5(a). Because the VB has a nonmonotonic dispersion with side maxima hosting a high density of states, the Fermi level $E_{F}$ must be pinned close to the energy of these side maxima on a large range of gate voltages. This naturally explains the anomalously large quantum plateau $N=-1$, which starts at a lower magnetic field and continues until a higher magnetic field than expected from a simple estimate of the positions of the filling factors $\nu=-1 / 2$ and $\nu=-3 / 2$ in the $\left(V_{g}, B\right)$ plane.

To get numerical estimates, we have calculated the dispersion of the LLs by using an 8 band $\mathbf{k} \cdot \mathbf{p}$ method [4]. The disorder is taken into account by introducing a constant Gaussian broadening $\Gamma \sim 5 \mathrm{meV}$ for each LL. The broadening was estimated from the damping of the Shubnikov-de Haas oscillations in the VB. The result is shown in Fig. 6. The LLs in the CB have a monotonic electron-like dispersion, roughly in the power of $B^{0.5}$. The two zero-mode LLs cross at $B \simeq 2 \mathrm{~T}$ and also have a monotonic, almost linear, dispersion. By contrast, the LLs in the VB have a nonmonotonic dispersion (for clarity, one of these LLs is shown in purple in Fig. 6). At low magnetic fields, when their eigenstates are localized in the center of the Brillouin zone, the LLs of the VB have a hole-like dispersion. At higher magnetic field (around $B \simeq 3 \mathrm{~T}$ for the LL enlightened in purple), when the LL eigenstates leave the center of the Brillouin zone and slip toward the side maxima, the LL dispersion changes its direction and becomes electron-like. Finally, at even larger $B$ when the eigenstates leave the side maxima, still increasing their momenta, the LLs retrieve a hole-like dispersion.

The upper LLs of the VB are energetically very close: the first upper 50 LLs lie in a $2 \mathrm{meV}$ energy range at $B=3 \mathrm{~T}$. As this range is smaller than $\Gamma$, all these LLs are partly populated simultaneously when $E_{F}$ is in the 


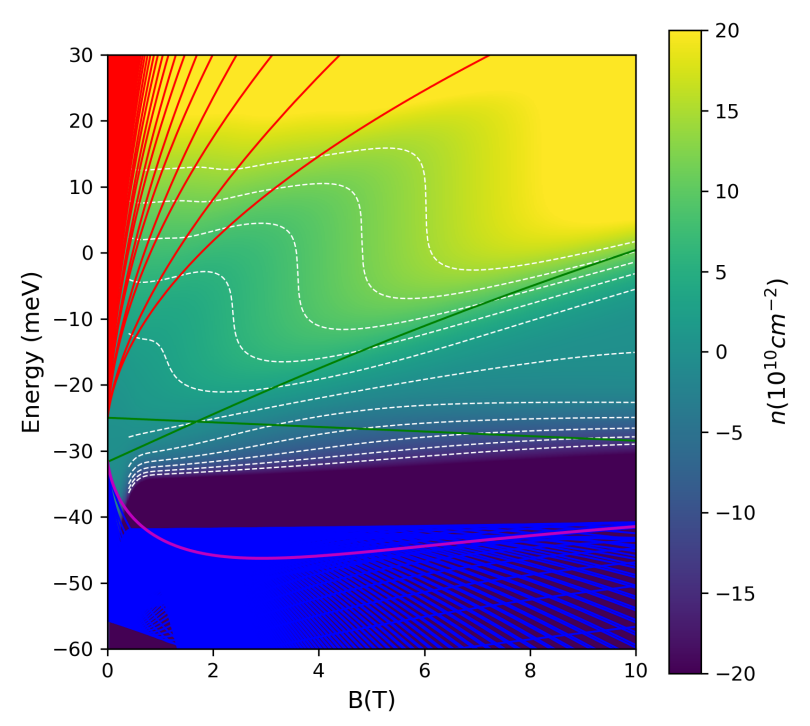

FIG. 6. Landau level in our sample at $T=260 \mathrm{mK}$ on the basis of the 8-band $\mathbf{k} \cdot \mathbf{p}$ Hamiltonian [4]. The LLs in the $\mathrm{CB}$ and $\mathrm{VB}$ are shown as red and blue lines, respectively. One of the LL of the VB is shown in violet to evidence its nonmonotonic dispersion. The two zero-mode LLs are shown as green solid lines. The chemical potential is indicated with white dashed lines for different values of the gate voltage, from 0 (upper curve) down to $-3 \mathrm{~V}$ (lowest curve) with a step of $-0.3 \mathrm{~V}$. A Gaussian broadening $\Gamma=5 \mathrm{meV}$ was added in the calculation. A color map of the total carrier concentration $n$ is shown in the background.

$N=-1$ plateau. This quasi-continuum of LLs acts as a charge reservoir. To check this, we evaluated the position of the Fermi energy $E_{F}$ as a function of $V_{g}$ and $B$. The result is shown in Fig. 6 for various values of $V_{g}$. In the CB, the Fermi energy $E_{F}$ oscillates between the LLs before collapsing down to the upper zero-mode LL. The magnetic field range of the $N=1$ plateau is relatively limited for all gate voltages. By contrast, in VB, $E_{F}$ is pinned in the $N=-1$ plateau over an extended magnetic field range. At $V_{g}=-2.5 \mathrm{~V}, E_{F}$ enters the $N=-1$ plateau at $B \simeq 0.5 \mathrm{~T}$ and is still in the gap at $B=3 \mathrm{~T}$. These findings are in agreement with the observed width of the QH plateau in Fig. 3.

\section{Model for the transconductance fluctuations}

The localization model presented before, based on the screening of the disorder, is valid when only one LL is partly populated, whereas all the other LLs are either empty or filled. This model predicts a unique slope $d V_{g} / d B=-e^{2} / h C_{g}$ on the $N=-1$ plateau. In the situation corresponding to Fig. 6, the screening is simultaneously induced by several partly populated LLs. The
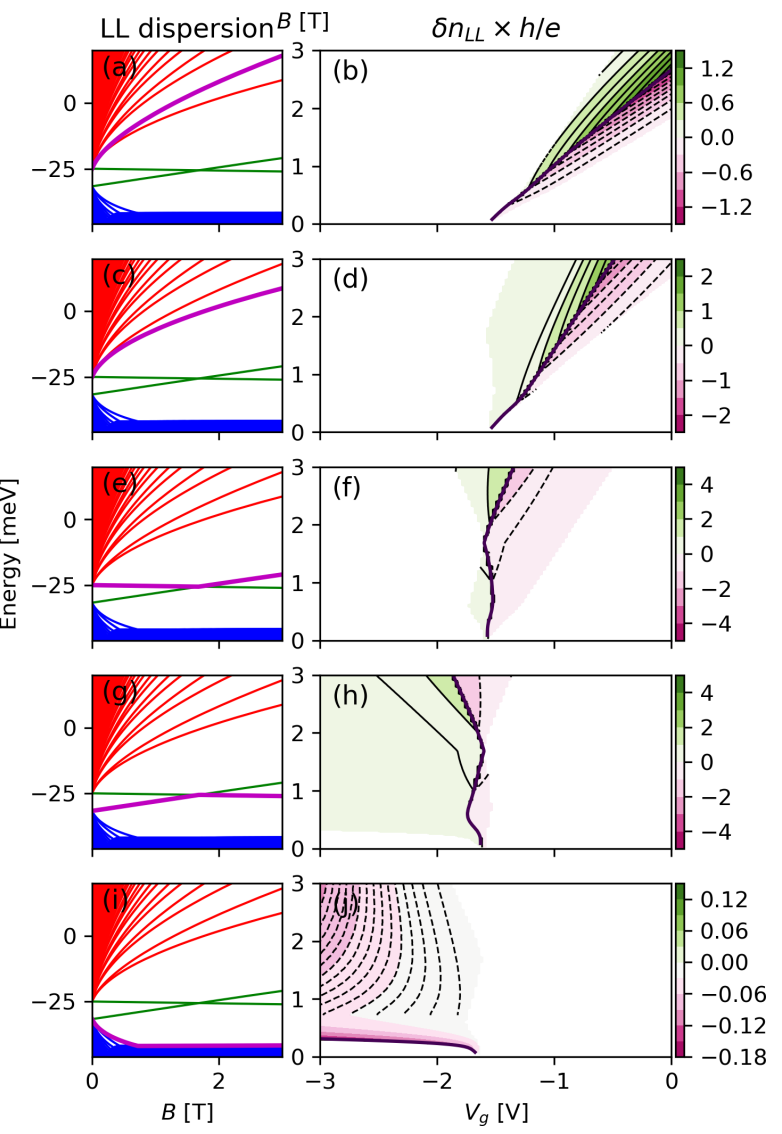

FIG. 7. (b,d,g,h,j) Color map in the $\left(V_{g}, B\right)$ plane rendering the isocharge lines $\delta n$ for the LLs indicated as thick magenta lines in panels a, c, e, g, and i, respectively. The solid and dashed lines are contour lines for positive and negative $\delta n$, respectively.

numerical calculation of the screened disorder in such a situation is outside the scope of this work [32]. The overall picture is, nevertheless, well established. When $E_{F}$ is in the $N=-1$ plateau, just above the VB, the 2DEG is an incompressible sea where $\nu=-1$, in which appear compressible antidots where $\nu<-1$. These antidots are themselves formed of a concentric succession of incompressible and compressible regions, which correspond to the progressive filling of the underlying LLs of the VB.

As the upper LL of the VB delimits the boundary between the incompressible sea and antidots, we assume that this peculiar LL is responsible for the most visible TFs. We extend the previous model assuming that the TFs are formed by the filling $\delta n_{u}$ of the upper LL of the VB only, while the lower LLs act only as a reservoir.

To sustain this hypothesis, we need to calculate the lines of constant $\delta n$ for each LL in the $\left(V_{g}, B\right)$ plane and check if these lines are deflected with respect to the initial model. In the following, we call isocharges these lines of constant $\delta n$ in the $\left(V_{g}, B\right)$ plane. First, one point is chosen in the $\left(V_{g}, B\right)$ plane, and the LL dispersion, density of states, electrochemical potential, and carrier density for each LL are calculated with the numerical model used to 


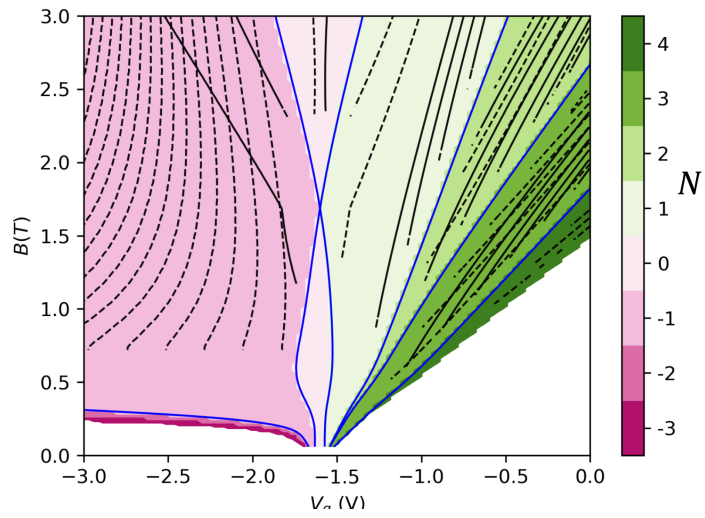

FIG. 8. Colormap in the $\left(V_{g}, B\right)$ plane, indicating the area corresponding to Chern numbers $N=-3, \ldots 4(N=$ $\left.-\sigma_{x y} h / e^{2}\right)$. The isocharge lines $\delta n$ for the 5 LLs detailed in Fig. 7 are also superimposed on this colormap.

calculate Fig. 6 with a macroscopic Gaussian broadening of the LLs. This operation is repeated for each point in the $\left(V_{g}, B\right)$ plane to separately retrieve the isocharges for each LL. The result is shown in Fig. 7. Figs. 7(b,d) show the isocharges for the second and first LLs of the CB. These LLs are enlightened in the left panels (a) and (c), respectively. The isocharges are separated into two groups whose slope corresponds to $\nu=2$ and 3 for the second LL (Fig. 7b) and $\nu=1$ and 2 for the first LL (Fig. 7d). The change of the slope takes place when the LL is half-filled. In the $\left(V_{g}, B\right)$ plane, this corresponds to lines corresponding to half integer filling factor $\nu=3 / 2$ and $\nu=5 / 2$. This is in remarkable agreement with both experimental data and the charge localization model when only one LL screens the disorder.

Similar results are reproduced for the two zero-mode LLs, as shown in Fig. 7(e-h). The isocharges again follow quantized slopes corresponding to $\nu=1,0$ (upper zeromode LL, Fig. $7(\mathrm{e}, \mathrm{f}))$ and $\nu=0,-1$ (lower zero-mode LL, Fig. $7(\mathrm{~g}, \mathrm{~h}))$. A slope deviation is predicted only in the vicinity of the crossing of the two zero-mode LLs around $B \simeq 2 \mathrm{~T}$.

The situation becomes more complex in the VB. Here, the isocharges for the upper LL of the VB are shown in Fig. $7(\mathrm{i}, \mathrm{j})$. These isocharges do not follow any quantized value, and have a strong nonmonotonic character. Below $B=1 \mathrm{~T}$, the model predicts isocharges with negative slopes, as observed experimentally. However, this is the limit of validity for the model because not enough LLs are taken into account (we calculate only the first 320 LLs of the VB). More interestingly, between $B=1$ and $B=2 \mathrm{~T}$, the slope of the isocharges changes its signs and becomes positive $\left(d V_{g} / d B>0\right)$. Finally, at higher $B$, the slope becomes almost vertical, $d V_{g} / d B \simeq 0$. This nonmonotonic behavior is qualitatively coincides with the experimental observations.

Furthermore, we calculated the isocharges for the first ten upper LLs of the VB, and for all, we found the same trend, i.e. all the isocharges have unusual positive slopes in the $N=-1$ plateau.

A hand waving explanation for the unusual behavior of these isocharges is as follows. At low $V_{g}$ values, $E_{F}$ is pinned on top of the $\mathrm{VB}$ and slightly varies with $B$, as seen in Fig. 6. Thus, the filling factor of the upper LL of the VB, $\nu_{u}$, does not depend on $B$ and is a monotonically increasing function of $V_{g}: \nu_{u}=f\left(V_{g}\right) \simeq$ $f(0)+f^{\prime}(0) V_{g}$. Using $\delta n_{u}=\left(\nu_{u}-1\right) e B / h$, it follows that $V_{g}=c^{t e}+f^{\prime}(0)^{-1} \delta n_{u}(h / e B)$, which gives a positive slope $\partial V_{g} / \partial B$ as $\delta n_{u}<0$ and $f^{\prime}(0)>0$. This hyperbolic behavior induces the nonmonotonic dispersion of the $\delta n_{u}$ isocharges.

\section{Discussion}

The different isocharges of the five LLs presented in Fig. 7 have been superimposed in Fig. 8 to present a more complete view of the predicted fluctuations. This model reproduces the quantization of the slope of the isocharges in the CB. Since the energy separation between the LLs in the CB is larger than LL broadening, only one LL is partly (de)populated.

Remarkably, the model reproduces many of the experimental features observed on the $N=-1$ plateau. In particular, TFs with positive and negative slopes are reproduced. Isocharges with a slope corresponding to $\nu=-1$, associated with the lower zero-mode LL, are predicted around $B \simeq 2.5 \mathrm{~T}, V_{g} \simeq-2 \mathrm{~V}$, as shown by the solid black lines in Fig. 8. The isocharges of the lower zeromode LL and the upper LL of the VB follow very distinct trajectories. The isocharges of the upper LL of the VB are shown as black dashed lines in the $\sigma_{x y}=-e^{2} / h$ region. The slopes of these isocharges are positive at low $B$ and become more vertical in the $\left(V_{g}, B\right)$ plane when $B$ increases, where they can even become slightly negative.

This roughly corresponds to what is observed experimentally where At an intermediate magnetic field $1.2 \mathrm{~T}$ $<B<3 \mathrm{~T}$, the TFs slopes are positive and increase when $V_{g}$ increases, whereas at higher magnetic fields, the TFs slopes are almost vertical in the $\left(V_{g}, B\right)$ plane.

Nevertheless, interesting discrepancies exist. On the $N=-1$ plateau, the TFs appear experimentally as sets of parallel lines with a constant slope, and we do not observe TFs with varying slopes, as predicted by the model. Experimentally, there is also the superposition of TFs with various slopes. This is well visible, for instance around $\left(V_{g}=-2.5 \mathrm{~V}, B=2 \mathrm{~T}\right)$, where three sets of TFs with slopes $\nu \simeq 5,1.5$ and 0 are superimposed. This suggests that microscopically, the 2DEG has been torn apart and regions of different local filling factors coexist at different places. These observations underline that the proposed model does not reproduce the microscopic features as all the complexity of charge localization, screening, and Coulomb blockade is hidden in the unique macroscopic parameter $\delta n$. 


\section{CONCLUSION}

To conclude, we conducted transconductance measurements on a macroscopic Hall bar made from an inverted HgTe quantum well. We observed pronounced and reproducible transconductance fluctuations in the $\left(B, V_{g}\right)$ plane due to the screening and charge localization. We conclude that transconductance measurements can reveal microscopic states, even when measuring macroscopic devices. In the $\mathrm{CB}$, the slopes of these TFs follow lines of positive integer filling factors, as already observed in other 2DEGs. However, in the VB, we observe an unexpected behavior, i.e. transconductance fluctuations with positive slopes. We attribute the appearance of these unexpected fluctuations to the charge localization in the quantum states $\nu=-1$ of the upper LL localized in the side maxima of the VB. The unusual slope of these fluctuations is attributed to the underlying large density of states in these side maxima, which acts as a charge reservoir and deflects the expected trajectories of the fluctuations.

\section{ACKNOWLEDGMENTS}

This work was supported by the CNRS via the IRP "TeraMIR," Montpellier University through the "Occitanie Terahertz Platform," the Russian Science Foundation (RSF-ANR) grant 20-42-09039, the French Agence Nationale pour la Recherche (Colector and Dirac3D projects), and the European Union (Flag-Era JTC 2019 - DeMeGras). Sample characterization was performed in the frame of the RSF-ANR Colector project. This project received funding from the European Union's Horizon 2020 research and innovation program under the Marie Skłodowska-Curie grant agreement no. 765426 (TeraApps). We thank F. Geniet and C. Lhenoret for fruitful discussions.
[1] B. A. Bernevig, T. L. Hughes, and S.-C. Zhang, Science 314, 1757 (2006).

[2] M. Konig, S. Wiedmann, C. Brune, A. Roth, H. Buhmann, L. W. Molenkamp, X.-L. Qi, and S.-C. Zhang, Science 318, 766 (2007).

[3] A. M. Kadykov, S. S. Krishtopenko, B. Jouault, W. Desrat, W. Knap, S. Ruffenach, C. Consejo, J. Torres, S. V. Morozov, N. N. Mikhailov, et al., Phys. Rev. Lett. 120, 086401 (2018).

[4] S. S. Krishtopenko, I. Yahniuk, D. B. But, V. I. Gavrilenko, W. Knap, and F. Teppe, Physical Review B 94, 245402 (2016).

[5] G. Landwehr, J. Gerschütz, S. Oehling, A. PfeufferJeschke, V. Latussek, and C. R. Becker, Physica E 6, 713 (2000).

[6] K. Ortner, X. C. Zhang, A. Pfeuffer-Jeschke, C. R. Becker, G. Landwehr, and L. W. Molenkamp, Phys. Rev. B 66, 075322 (2002).

[7] Z. D. Kvon, E. B. Olshanetsky, E. G. Novik, D. A. Kozlov, N. N. Mikhailov, I. O. Parm, and S. A. Dvoretsky, Phys. Rev. B 83, 193304 (2011).

[8] G. M. Minkov, A. V. Germanenko, O. E. Rut, A. A. Sherstobitov, S. A. Dvoretski, and N. N. Mikhailov, Phys. Rev. B 88, 155306 (2013).

[9] G. M. Minkov, A. V. Germanenko, O. E. Rut, A. A. Sherstobitov, S. A. Dvoretski, and N. N. Mikhailov, Phys. Rev. B 89, 165311 (2014).

[10] G. M. Minkov, A. V. Germanenko, O. E. Rut, A. A. Sherstobitov, M. O. Nestoklon, S. A. Dvoretski, and N. N. Mikhailov, Phys. Rev. B 93, 155304 (2016).

[11] I. Yahniuk, S. S. Krishtopenko, G. Grabecki, B. Jouault, C. Consejo, W. Desrat, M. Majewicz, A. M. Kadykov, K. E. Spirin, V. I. Gavrilenko, et al., npj Quantum Materials 4, 13 (2019).

[12] W. Zawadzki, A. Raymond, and M. Kubisa, Physica Status Solidi (b) 251, 247 (2014).
[13] S. Kopylov, A. Tzalenchuk, S. Kubatkin, and V. I. Fal'ko, Appl. Phys. Lett. 97, 112109 (2010).

[14] T. J. B. M. Janssen, A. Tzalenchuk, R. Yakimova, S. Kubatkin, S. Lara-Avila, S. Kopylov, and V. I. Fal'ko, Phys. Rev. B 83, 233402 (2011).

[15] M. Yang, O. Couturaud, W. Desrat, C. Consejo, D. Kazazis, R. Yakimova, M. Syväjärvi, M. Goiran, J. Béard, P. Frings, et al., Phys. Rev. Lett. 117, 237702 (2016).

[16] D. S. Lee, V. Skákalová, R. T. Weitz, K. von Klitzing, and J. H. Smet, Phys. Rev. Lett. 109, 056602 (2012).

[17] Y. Kim, D. S. Lee, S. Jung, V. Skákalová, T. Taniguchi, K. Watanabe, J. S. Kim, and J. H. Smet, Nano Lett. 15, 7445 (2015).

[18] N. N. Berchenko and M. V. Pashkovskii, Sov. Phys. Usp. 19, 462 (1976).

[19] A. M. Dykhne and I. M. Ruzin, Phys. Rev. B 50, 2369 (1994).

[20] B. Huckestein, Rev. Mod. Phys. 67, 357 (1995).

[21] Z. D. Kvon, E. B. Olshanetsky, N. N. Mikhailov, and D. A. Kozlov, Low Temp. Phys. 35, 6 (2009).

[22] D. H. Cobden, C. H. W. Barnes, and C. J. B. Ford, Phys. Rev. Lett. 82, 4695 (1999).

[23] S. Branchaud, A. Kam, P. Zawadzki, F. M. Peeters, and A. S. Sachrajda, Phys. Rev. B 81, 121406(R) (2010).

[24] J. Velasco, G. Liu, L. Jing, P. Kratz, H. Zhang, W. Bao, M. Bockrath, and C. N. Lau, Phys. Rev. B 81, 121407(R) (2010).

[25] S. Ilani, J. Martin, E. Teitelbaum, J. Smet, D. Mahalu, V. Umansky, and A. Yacoby, Nature 427, 328 (2004).

[26] J. Martin, N. Akerman, G. Ulbricht, T. Lohmann, K. Von Klitzing, J. Smet, and A. Yacoby, Nat. Phys. 5, 669 (2009).

[27] D. Kozlov, Z. Kvon, N. Mikhailov, and S. Dvoretsky, JETP Lett. 96, 730 (2013).

[28] E. Olshanetsky, Z. Kvon, M. Entin, L. Magarill, N. Mikhailov, I. Parm, and S. Dvoretsky, JETP Lett. 
89, 290 (2009).

[29] W. G. Baber, Proceedings of the Royal Society of London. Series A - Mathematical and Physical Sciences 158, 383 (1937).

[30] V. F. Gantmakher and Y. B. Levinson, Carrier Scattering in Metals and Semiconductors (Elsevier, 1987).
[31] J. A. Alexander-Webber, J. Huang, D. K. Maude, T. J. B. M. Janssen, A. Tzalenchuk, V. Antonov, T. Yager, S. Lara-Avila, S. Kubatkin, R. Yakimova, et al., Sci. Rep. 6, 30296 (2016).

[32] A. L. Efros, F. G. Pikus, and V. G. Burnett, Phys. Rev. B 47, 2233 (1993). 